\title{
Reducing Dose for Digital Cranial Radiography: The Increased Source to the Image-receptor Distance Approach
}

\author{
Maria Joyce, $\mathrm{PhD}^{\mathrm{ab} *}$, Mark McEntee, $\mathrm{PhD}^{\mathrm{b}}$, Patrick C. Brennan, $\mathrm{PhD}^{\mathrm{b}}$ and Desiree O'Leary, $\mathrm{PhD}^{\mathrm{a}}$ \\ ${ }^{a}$ The School of Medicine and Medical Science, University College Dublin, Belfield, Dublin, Ireland \\ ${ }^{\mathrm{b}}$ Discipline of Medical Radiation Sciences, University of Sydney, Lidcombe, NSW, Australia
}

\begin{abstract}
This investigation proposes that an increased source to the imagereceptor distance (SID) technique can be used to optimize occipital frontal and lateral cranial radiographs acquired with direct digital radiography. Although cranial radiography is not performed on a routine basis, it should nonetheless be optimized to keep the dose to the patient as low as reasonably achievable, particularly because it can form part of the facial bone and sinus series. Dose measurements were acquired at various SIDs, and image quality was assessed using visual grading analysis. Statistically significant reductions in the effective dose between $19.2 \%$ and $23.9 \%$ were obtained when the SID was increased from the standard 100 to $150 \mathrm{~cm}(P \leq .05)$, and visual grading analysis scores indicate that image quality remained diagnostically acceptable for both projections. This investigation concludes that increasing the SID effectively optimizes occipital frontal and lateral skull radiographs. Radiology departments must be advised of the benefits of this technique with the goal of introducing an updated reference SID of $150 \mathrm{~cm}$ into clinical practice.
\end{abstract}

\section{Introduction}

When considering the optimization of medical exposures, it is crucial that the radiation dose to the patient is kept as low as reasonably achievable while ensuring that image quality is sufficient for diagnostic purposes $[1,2]$. The importance of regularly investigating optimization strategies and adhering to the best radiographic practice is highlighted by advancements in current dose reduction literature and the steady increase in public concern regarding the biological effects of

\footnotetext{
Supported by the University College Dublin Ad Astra Scholarship programme.

* Corresponding author: Maria Joyce, PhD, M Block - Room M206, The University of Sydney-Cumberland Campus, 75 East Street, Lidcombe, NSW 2141, Australia.

E-mail address: maria.joyce@sydney.edu.au (M. Joyce).
}

\begin{abstract}
RÉSUMÉ
Cette étude pose comme hypothèse que la technique de l'augmentation de la distance entre la source et le récepteur d'image (SID) peut être utilisée pour optimiser les radiographies occipitales frontales $\left(\mathrm{OF} 10^{\circ}\right)$ et crâniales latérales acquises par radiographie numérique directe. Bien que la radiographie crâniale ne se pratique pas couramment, elle doit quand même être optimisée pour garder la dose pour le patient la plus basse qui soir raisonnablement possible d'atteindre, notamment parce qu'elle peut s'inscrire dans une série de la structure osseuse faciale et des sinus. Des mesures de dose ont été prises à différentes SID et la qualité de l'image a été évaluée au moyen de l'analyse de classement visuel (VGA). Des réductions statistiquement significatives de la dose variant entre $19,2 \%$ et $23,9 \%$ ont été obtenues lorsque la SID a été portée de la distance standard de $100 \mathrm{~cm}$ à $150 \mathrm{~cm}(\mathrm{p} \leq 0,05)$ et les notes VGA indiquent que la qualité diagnostique de l'image reste acceptable pour les deux projections. Cette étude conclut que l'augmentation de la SID permet effectivement d'optimiser les radiographies occipitales frontales et crâniales latérales. Les services de radiographie doivent être informés des avantages de cette technique dans le but d'adopter une mise à jour à $150 \mathrm{~cm}$ de la SID de référence dans la pratique clinique.
\end{abstract}

low-level ionizing radiation [3]. Uffmann and SchaeferProkop [4] maintain that throughout the transition from film screen to digital technology in the clinical environment many of the examination parameters such as tube current $(\mathrm{mA})$, tube voltage $(\mathrm{kVp})$, and filtration were translated directly from film screen exposures to computed radiography and direct digital radiography $(\mathrm{dDR})$ without appropriate radiographic adjustment [4]. A review of the available optimization techniques is required so that the evidence base for optimized radiographic practice is increased and ongoing dose reductions with digital acquisition devices are achieved [5]. One such optimization technique is increasing the source to image-receptor distance (SID) for $\mathrm{x}$-ray examinations within direct digital radiography.

Increasing the SID for radiographic examinations has proven results for film screen, computed radiography, and 
dDR technology [6-10], yet despite this evidence base, anecdotal evidence and teaching practice show that it is not commonly practiced within radiology departments. Previous work established that increasing the SID had the advantage of being a low-cost method of dose reduction that did not negatively impact patient comfort or image quality [8]. However, a review of the literature revealed that the research does not cover a broad range of radiographic examinations; seven studies researched anterior-posterior (AP) pelvis examinations [5,8-13], four studies researched AP and lateral lumbar spine examinations $[5,6,11,12]$, one study researched AP abdomen examinations [12], and one study researched AP knee examinations [7]. In addition, the level of effective dose reduction varied among authors, ranging from $8.1 \%$ for computer-simulated calculations [14] to a maximum of $44 \%$ for patient studies [6].

The current investigation proposes that the increased SID technique can be used as an optimization tool for cranial radiography. A review of the private sector in Australia revealed that from June 2011 to June 2012 a total of $1.1 \%$ of all diagnostic radiology examinations conducted in that year were cranial examinations, which include skull projections, facial bones, and sinuses [15]. Imaging of head injury is normally managed by following clinical guidelines or decision support tools such as the National Institute for Health and Clinical Excellence Head Injury Guidelines [16] or the Canadian CT Head Rule and the New Orleans Criteria [17]. These guidelines suggest the appropriate diagnostic imaging pathway for each type of head injury, and even though projection radiography is not a recommended imaging modality, it is still performed in some countries and has value in specific diagnostic situations [18]. It can be used to confirm a clinical suspicion of fracture or to locate metallic foreign objects embedded in the skull, and it can comprise part of a skeletal survey for multiple myeloma [19].

Although skull radiography is performed less frequently in the developed world (North America, Canada, Europe, and Australia), it is still performed in many developing countries because of the costs associated with computed tomographic imaging. A study reviewing radiology departments in Sao Paulo, Brazil, reported that head (skull and sinus) examinations are one of the three most frequent examinations performed at a rate of 47 per 1,000 population, and with a population of 39.2 million this accounts for 1.84 million people annually [20]. Given the more frequent usage in developing countries and its occasional usage in developed countries, every effort should be made to ensure that all cranial projections are optimized to keep the dose to the patient as low as reasonably achievable, particularly because some patients will most likely undergo subsequent computed tomographic imaging $[16,17,21]$.

Evidence shows that increasing the SID from 100 to $130 \mathrm{~cm}$ can optimize lumbar spine, pelvic, abdominal, and lower-extremity examinations [5-13] although no literature is currently found for patient and/or phantom use of an increased SID for skull examination. Poletti and McLean
[14] produced Monte Carlo calculations and showed that increasing the SID to $200 \mathrm{~cm}$ resulted in a reduction in the effective dose of $35.4 \%$ for postero-anterior (PA) skull projection and $34.3 \%$ for lateral skull projection when the collimation borders were simulated to the outer surface of the skull at each SID. Since the work by Poletti and McLean, the MonteCarlo simulation package PCXMC has been updated to version 2.0, which facilitates the assessment of the exposureinduced cancer risk, thus providing a better indication of how an increased SID affects the patient.

The verification of theory with direct experimental investigations is crucial to the clinical implementation of any new optimization technique $[8,14]$; thus, the current investigation aimed to establish the efficacy of the increased SID technique for two specific cranial examinations using a dDR system. The projections examined were the occipital frontal $\left(\mathrm{OF} 10^{\circ}\right)$ and the lateral cranial projections, which are the more frequently performed examinations and part of facial bone and sinus series as well. These examinations lend themselves to the increased SID technique because both the $\mathrm{OF} 10^{\circ}$ and lateral cranial projections can be performed with a horizontal $\mathrm{x}$-ray beam in clinical practice [22] and do not require additional bending or stretching by the radiographer because the tube would be travelling in the horizontal plane.

\section{Methodology}

The experimental protocol for both projections involved the collection of dosimetry data at increased increments of SID and an image assessment of the resultant radiographs to establish the efficacy of an increased SID as an optimization technique for this type of examination.

\section{Radiographic Technique}

\section{OF10 Cranial Projection}

The SK100 skull phantom (The Phantom Laboratory, Salem, NY) was positioned with the nose and forehead touching the image detector and the orbital-meatal baseline at $10^{\circ}$ to the detector. This angulation is known as an OF $10^{\circ}$ caudal skull projection or the Caldwell projection $[23,24]$. The median-sagittal plane was aligned perpendicular to the image receptor in line with the central $x$-ray beam. The central ray exit point was through the nasion, and the skull was angled so that the petrous ridge was in the middle third of the orbits [23]. The $\mathrm{x}$-ray tube head was horizontal at an angle of $90^{\circ}$ to the image receptor, simulating the patient in the erect position as per the usual clinical protocol for skull image acquisition [23]. The SIDs under investigation were 100 (routine clinical SID), 130, and $150 \mathrm{~cm}$. Collimation was to the outer surface of the phantom laterally and to the vertex of the skull superiorly at each SID. Five exposures were acquired per SID at $75 \mathrm{kVp}$ [24] using the central chamber of the automatic exposure device (AED) [24, 25]. When using the $\mathrm{AED}$, the $\mathrm{mA}$ values were automatically controlled, and these values increased as the SID increased. In clinical practice, the $\mathrm{OF} 10^{\circ}$ skull projection requires the 
use of a radiographic grid, and because the only grids available to the research facility were focused to distances of 100 and $180 \mathrm{~cm}$, the radiographs acquired at an SID of 100 or $130 \mathrm{~cm}$ used the $100-\mathrm{cm}$ grid (focal range of $86-120 \mathrm{~cm}$ ) and the radiographs acquired at an SID of $150 \mathrm{~cm}$ used the $180-\mathrm{cm}$ grid (focal range of $152-183 \mathrm{~cm}$ ). The rationale for using these grids is explored in the discussion section of this article. The imaging parameters used in the current methodology provided the optimal imaging conditions for the given experimental setup, and the tube voltage selection corresponds to values from a survey of clinical departments in the area. After exposure, all images were exported to the picture archiving and communication system workstation for evaluation (GE HealthCare, General Electric Company, Fairfield, CT).

\section{Lateral Cranial Projection}

The SK100 skull phantom was placed in the lateral position with the median-sagittal plane parallel to the image receptor, and the central $\mathrm{x}$-ray beam was directed to a point midway between the glabella and the external occipital protuberance approximately $2.5 \mathrm{~cm}$ above and in front of the internal auditory meatus [23]. The x-ray tube head was horizontal at an angle of $90^{\circ}$ to the image receptor, simulating positioning of the patient in clinical practice with the $\mathrm{x}$-ray beam in the horizontal position [22]. Collimation was to the external occipital protuberance laterally and to the vertex of the skull superiorly for each SID setting. The SIDs under investigation were 100,130 , and $150 \mathrm{~cm}$, and five exposures were acquired per SID at $70 \mathrm{kVp}$ [24] using the central chamber of the AED device $[24,25]$ and exported to the picture archiving and communication system for review. As with the $\mathrm{OF} 10^{\circ}$ projection, the lateral radiographs acquired at an SID of 100 or $130 \mathrm{~cm}$ used the $100-\mathrm{cm}$ grid, and the radiographs acquired at a SID of $150 \mathrm{~cm}$ used the $180-\mathrm{cm}$ grid.

\section{Dosimetry Data Collection}

There were four areas of dosimetry data collection for both projections: the central $\mathrm{x}$-ray beam entrance surface dose (ESD), the thyroid area, the lens of the eye (left), and the centre point of the image receptor (in front of the AED). Using the ESD measurements and the Monte Carlo simulation package PCXMC (version 2.0), the effective dose per exposure was calculated for each SID setting. This software package allows greater flexibility compared with dose coefficients published by the National Radiological Protection Board because the SID is taken into account in the calculation [26]. The radiation dose to the thyroid and eye was measured because these are radiosensitive areas within the collimation field, and the radiation dose at the image receptor was monitored to ensure that the same exposure was incident on the image receptor for all SIDs. A radiolucent sachet containing six preannealed thermoluminescent dosimeters (TLDs) was positioned on each site for the three SID settings and exposed 10 times to ensure sufficient radiation exposure of all TLDs [27]. The TLDs were read within 24 hours of exposure
[28], and the resulting data were divided by 10 to account for one exposure. Six TLDs were taken randomly from each batch of 100 TLDs and reserved as controls [6]. A multipurpose analyser acted as a secondary calibration tool for calibrating the TLDs and facilitated the conversion of the TLD readings from nanocoulombs to milligrays (mGy).

\section{Image Assessment}

The assessment of all images was performed by an evaluation panel of four radiographers with a mean experience level of 7.8 years in general radiography (standard deviation = 3.8 years). All radiographs were assessed using relative visual grading analysis (VGA) with a four-point scoring scale [29-31] as shown in Table 1. The inclusion of radiographers in this study was considered suitable because the observers were only required to assess the visibility of key anatomic landmarks and important image details within each image, and as such, specialist training was not required. This type of image assessment is performed by radiographers on a daily basis in the clinical setting. A review of the literature revealed that the composition of VGA evaluation panels does not follow a consistent pattern, with both radiologists and radiographers participating as observers $[6,8,32]$. In the current work, the observers first scored the OF $10^{\circ}$ skull images and then the lateral skull images. There were 15 test images for each projection and one reference image (either $\mathrm{OF} 10^{\circ}$ or lateral), which was taken at an SID of $100 \mathrm{~cm}$ and showed adequate visualization of all criteria [8]. The images were viewed in pairs on adjacent monitors with the radiographic reference image displayed on the right and randomized test images displayed consecutively on the left. The observers were blinded to the acquisition parameters and thus were unaware that the radiographs were acquired at different SIDs. The test images were coded using numbers one through 15 and arranged in random order to reduce bias [33]. Image viewing conditions remained constant throughout the investigation [34].

All images were assessed using modified diagnostic requirements from the European Guidelines on Quality Criteria for Radiographic Skull Images [25] as shown in Table 2. Before the evaluation process, each observer was given a set of instructions to ensure consistent interpretation of the quality criteria on each assessment form. Each observer completed an image quality assessment form for each of the OF $10^{\circ}$ and lateral skull radiographs.

\begin{tabular}{|c|c|}
\hline Score & Image Scoring Definitions \\
\hline 0 & $\begin{array}{l}\text { Poor; anatomy visualized worse than the reference image and } \\
\text { unacceptable }\end{array}$ \\
\hline 1 & $\begin{array}{l}\text { Acceptable; anatomy visualized worse than reference image but } \\
\text { acceptable }\end{array}$ \\
\hline 2 & Optimum; anatomy visualized equal to the reference image \\
\hline 3 & Excellent; anatomy visualized better than reference image \\
\hline
\end{tabular}

Table 1

Four-point Scoring Scale Used to Assess the Quality of Each Radiograph 
Table 2

Quality Criteria Used to Assess the OF10 ${ }^{\circ}$ and Lateral Skull Radiographs

$\mathrm{OF} 10^{\circ}$ skull projection

1. Reproduction of the lambda

2. Visually sharp reproduction of the frontal sinus, ethmoid cells, and apex of the petrous temporal bones and the internal auditory canals

3. Reproduction of the cochlear canals

4. Visually sharp reproduction of the outer and inner lamina of the cranial vault

Lateral skull projection

1. Visually sharp reproduction of the outer and inner lamina of the cranial vault, the floor of the sella, and the apex of the petrous temporal bone

2. Visually sharp reproduction of the vascular channels, the vertex of the skull, and the trabecular structure of the cranium

3. Reproduction of the posterior meningeal artery indentation

4. Reproduction of the suture of the squamous temporal bone

\section{Data Analysis}

Dosimetry data were tested for normality using the D'Agostino-Pearson normality test, and, subsequently, oneway analysis of variance (ANOVA) was performed on each dataset using a significance level of $P \leq .05[6,8]$. The post hoc analysis used in conjunction with ANOVA was the Tukey single-step multiple comparison test, which compares all possible pairs of means and is similar to the Student $t$ test except that it corrects for the probability of making a type I (false-positive) error by ensuring that the total error rate does not go above $\alpha=0.05$ [35]. The image quality data were categorized as ordinal nonparametric data, and the statistical test chosen to analyse the VGA scores was the KruskalWallis test using a significance level of $P \leq .05$. In order to control for the type I error, a Bonferroni adjustment, which is a multiple comparison correction, was applied to the data by multiplying the $P$ value by the number of SID comparisons made [35]. The inter- and intraobserver variability was expressed as the Fleiss kappa and Cohen kappa, respectively.

\section{Results}

\section{Dosimetry Data Analysis}

The dosimetry data revealed that increasing the SID from $100 \mathrm{~cm}$ results in a reduction in the radiation dose for OF $10^{\circ}$ and lateral cranial radiographs acquired using a dDR system. The effective dose for each exposure was calculated using the ESD values (which can be found in Figure 1) and the Monte Carlo simulation computer package PCXMC (version 2). Statistically significant reductions in the effective dose $(P \leq$ $.05)$ of $21.9 \%$ and $20.1 \%$ were obtained at the 150 - and $130-\mathrm{cm}$ respective SIDs when the imaging distance was increased from $100 \mathrm{~cm}$ for the $\mathrm{OF} 10^{\circ}$ skull examination (mean effective dose for $100 \mathrm{~cm}=0.0231 \mathrm{mSv}, 130 \mathrm{~cm}=$ $0.0183 \mathrm{mSv}, 150 \mathrm{~cm}=0.0178 \mathrm{mSv}$ ). Similarly, statistically significant reductions in the effective dose $(P \leq .05)$ of $23.9 \%$ and $19.2 \%$ were obtained for the lateral skull radiographs at the $150-$ and $130-\mathrm{cm}$ respective SIDs when compared with the 100-cm SID (mean effective dose for
Entrance surface dose at each SID for both projections

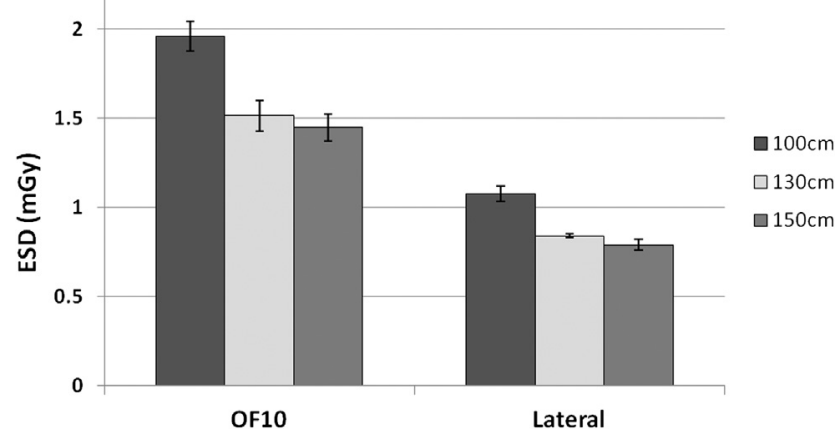

Figure 1. The mean ESD measurements (acquired using thermoluminescent dosimeters) at each SID for both the OF10 and lateral skull projections; standard deviations are shown as error bars.

$100 \mathrm{~cm}=0.0114 \mathrm{mSv}, 130 \mathrm{~cm}=0.0092 \mathrm{mSv}, 150 \mathrm{~cm}=$ $0.0087 \mathrm{mSv}$ ). In both cases, no statistically significant difference in the effective dose was observed between the 130- and 150 -cm data $(P>.05)$.

Analysis of the dosimetry data for the thyroid area revealed that increasing the SID from $100 \mathrm{~cm}$ significantly reduced the radiation dose to this radiosensitive area. Statistically significant reductions in the radiation dose of $39.5 \%$ and $34.0 \%$ were obtained for the $\mathrm{OF} 10^{\circ}$ skull radiographs, and reductions of $37.7 \%$ and $21.6 \%$ were obtained for the lateral skull radiographs at the 150 - and $130-\mathrm{cm}$ respective SIDs when compared with the $100-\mathrm{cm}$ SID $(P \leq .05)$. A comparison of the 130- and $150-\mathrm{cm}$ dosimetry data only produced a significant difference for the lateral skull examination $(P \leq .05)$, with a reduction of $20.6 \%$ achievable at the higher SID. Dosimetry measurements were also acquired for each projection to represent the radiation dose incident on the outer surface of the left eye at each SID. Analysis of these measurements for the OF $10^{\circ}$ skull radiographs found no statistically significant difference in radiation dose among the three SIDs $(P>.05)$. However, the dosimetry measurements for the lateral projection were found to differ significantly across the three imaging distances $(P \leq .001)$. Reductions in the radiation dose of $30.0 \%$ and $14.0 \%$ were obtained when the SID was positioned at 150 and $130 \mathrm{~cm}$, respectively, compared with $100 \mathrm{~cm}$. A statistically significant reduction in dose of $18.5 \%$ was also found when the SID was increased from 130 to $150 \mathrm{~cm}(P \leq .05)$.

The amount of radiation incident on the image receptor during the $\mathrm{OF} 10^{\circ}$ radiograph and the lateral skull radiographs was recorded throughout the study to ensure that the optical density was maintained at each SID. Analysis of these measurements using one-way ANOVA showed that there was no statistically significant difference among the imagereceptor doses at the three distances $(P>.05)$ for either projection, showing that the requirement of exposure consistency at the image receptor was upheld. Figure 2 gives a summary of the significant percentages of dose reductions only $(P \leq .05)$ for each SID comparison for both the OF $10^{\circ}$ and lateral skull projections. This graph shows that the ESD is also reduced by 


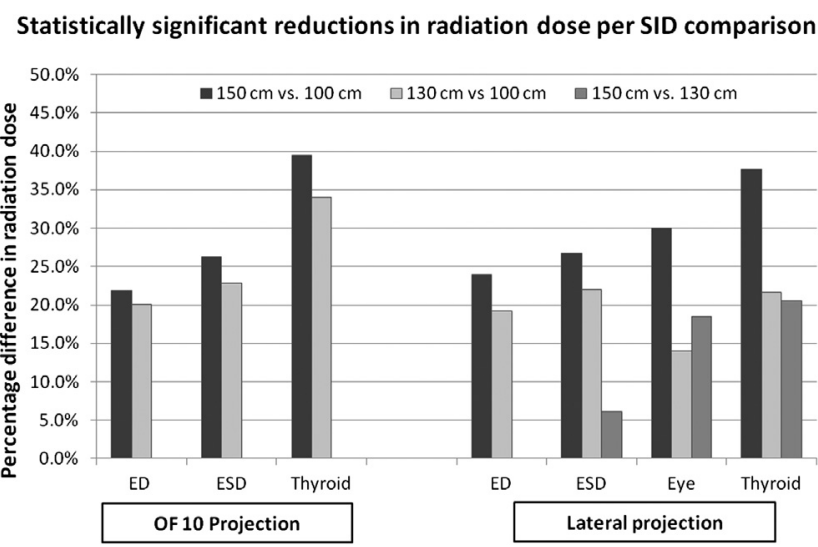

Figure 2. Summary of the significant percentages of dose reductions $(P \leq .05)$ for each SID comparison in which a positive percentage difference indicates a dose reduction at the higher SID. ED, effective dose.

an increased SID; however, it overestimates the reduction compared with the effective dose.

\section{Image Quality Assessment}

Analysis of the VGA scores for the $\mathrm{OF} 10^{\circ}$ skull radiographs showed that there was no statistically significant difference in image quality for radiographs acquired at each of the three SIDs $(P>.05)$. Analysis of the VGA scores for the lateral skull radiographs found a statistically significant decrease in the quality of radiographs acquired at 150 and $130 \mathrm{~cm}$ compared with $100 \mathrm{~cm}(P \leq .05)$; however, these images were still rated as diagnostically acceptable. Each of the individual quality criteria listed on the image quality assessment forms were analysed individually for each projection, and for the $\mathrm{OF} 10^{\circ}$ images a statistically significant decrease was observed in only one of the four imaging criteria (reproduction of the cochlear canals, Table 2) between the 100- and 150-cm images and the 130and $150-\mathrm{cm}$ images $(P \leq .05)$. Similarly, analysis of the individual quality criteria scores of the lateral skull radiographs revealed a statistically significant decrease in only one of the four imaging criteria (reproduction of the posterior meningeal artery indentation, Table 2$)$ between the 100 - and $130-\mathrm{cm}$ images and the 100- and $150-\mathrm{cm}$ images $(P \leq .001)$. Irrespective of this, all images were rated as diagnostically acceptable and showed adequate visualization of all criteria.

\section{Inter- and Intraobserver Variability}

Analysis of interobserver variability for the $\mathrm{OF} 10^{\circ}$ skull radiographs established fair agreement for three of the four observers (Fleiss kappa values ranging from 0.22 to 0.38 ) but poor agreement (Fleiss kappa $<0.20$ ) for one observer (observer 3). Similarly, for the lateral skull radiographs, interobserver agreement was fair for three of the four observers but poor for one observer (observer 2). Intraobserver agreement was found to be fair to moderate for three of the four observers for both projections (Cohen kappa values ranging from 0.36 to 0.48 ), with one observer (observer 4) showing poor agreement (Cohen kappa $<0.20$ ).

\section{Discussion}

The importance of verifying theoretical studies with experimental investigations is highlighted in the literature $[8,14]$, and the current work shows that for $\mathrm{dDR}$ skull examinations, increasing the SID is an effective method of reducing the radiation dose. Increasing the SID from $100 \mathrm{~cm}$ resulted in effective dose reductions between $19.2 \%$ and $23.9 \%$, and VGA scores indicate that image quality was still rated as adequate for both projections. The dosimetry results are in keeping with that of Poletti and McLean [14], who used computer simulation to estimate effective dose reductions of around 35\% for these two projections at an increased SID of $200 \mathrm{~cm}$. However, a direct comparison cannot be made because the work does not state the estimated dose reduction at an SID of $150 \mathrm{~cm}$ for this projection. The literature postulates that for certain examinations, increasing the SID can reduce geometric unsharpness and magnification in images $[8,36]$. In relation to the lateral skull radiograph (Figure 3), this would assist in the superimposition of bony structures such as the wings of the sphenoids and the internal auditory
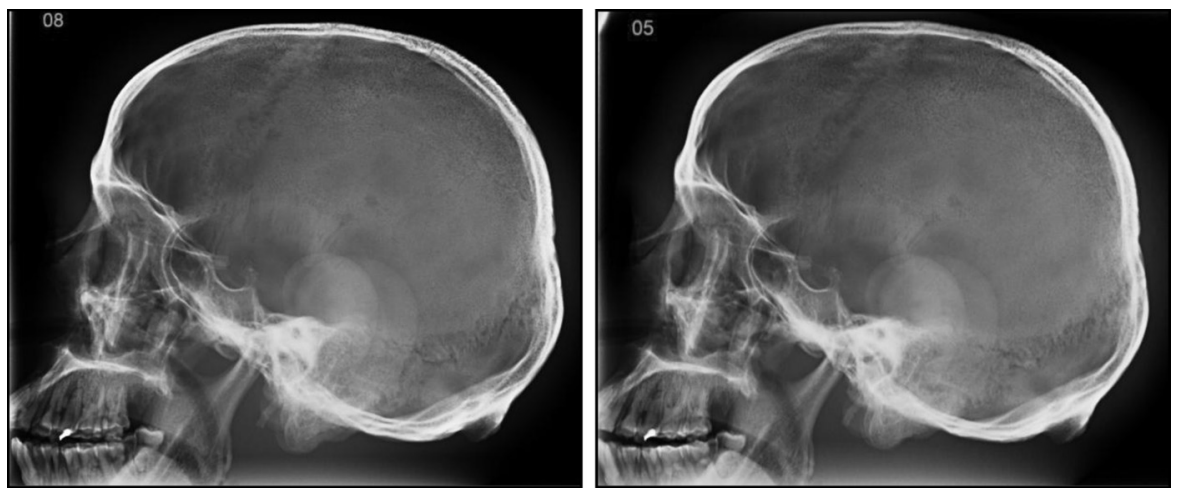

Figure 3. An example of lateral skull radiographs acquired at an SID of $100 \mathrm{~cm}$ (numbered 08) and $150 \mathrm{~cm}$ (numbered 05). Although the 100-cm images scored a higher VGA score, the 150-cm images were still considered visually acceptable in accordance with the CEC guidelines [25]. The reduction in the effective dose for the radiograph acquired at $150 \mathrm{~cm}$ was $37.7 \%$. Improved superimposition of several image features, such as wings of the sphenoids and the internal auditory meati, are noted with an increased SID. 
meati. The effect of increasing the object to source distance reduces the apparent object to image-receptor distance, with a resultant increase in the image quality with regard to patient positioning [36]. Although the current investigation did not assess patient positioning, this element of an increased SID is an advantage to radiographers positioning less cooperative patients and patients who cannot achieve paralleling of the median sagittal plane to the image receptor.

Although the $100-\mathrm{cm}$ images scored a higher VGA score, the $150-\mathrm{cm}$ images were still considered visually acceptable in accordance with the Commission of the European Communities (CEC) guidelines [25]. The reduction in the effective dose for the radiograph acquired at $150 \mathrm{~cm}$ was $37.7 \%$. Improved superimposition of several image features, such as wings of the sphenoids and the internal auditory meati, are observed with an increased SID.

With this investigation, the question of diagnostic acceptability is of importance. What is an acceptable level of image quality for diagnosis, as opposed to what is an aesthetically acceptable image to a radiographer or radiologist? The results of this study show that although the $100-\mathrm{cm}$ images scored the highest, increasing the SID does not reduce the radiographs to a level where the quality of the image is deemed unacceptable. An example of this is in the visualization of the meningeal artery indentation. This indentation appears as a subtle detail on the lateral skull radiograph, but as a fracture is double the depth of this indentation [37]; therefore, it is apparent that even poorly rated or slight visualization of the arterial indentation would result in acceptable visualization of a fracture. The results of the image quality evaluation in the current work revealed a statistically significant difference for one out of the four imaging criteria (reproduction of the cochlear canals) for the $\mathrm{OF} 10^{\circ}$ skull radiographs. A review of the individual VGA scores for these images found that although the $150-\mathrm{cm}$ images achieved a lower score than the reference image for this criterion, all anatomy within these images was still recognized as visually acceptable. This establishes that increasing the SID from 100 to $150 \mathrm{~cm}$ does not deteriorate the quality $\mathrm{OF} 10^{\circ}$ skull radiographs below acceptable levels because all necessary image quality criteria were visualized. For lateral skull radiographs, the $100-\mathrm{cm}$ images scored the highest in terms of image quality, but the visualization of particular anatomy on the 130- and $150-\mathrm{cm}$ images was still considered visually acceptable in accordance with the CEC guidelines and thus suitable for making clinical diagnoses [25]. The VGA scores were analysed for intraobserver variability, which revealed that for one observer (observer 4) there was variability in image scoring across all SIDs. By reanalysing the data without the VGA scores that contained intraobserver variability, the only statistically significant difference observed was between the 100- and $150-\mathrm{cm}$ images with the shorter SID displaying superior quality. A consideration for future VGA studies would be to provide a suitable training session before evaluation, which would ensure that no ambiguity exists among observers, or to provide a gold standard image with the areas marked therein.
An acknowledged limitation of the current work is that the 130 - and $150-\mathrm{cm}$ images were acquired with radiographic grids that were not focused to each specific SID. When considering the effect that grid cutoff (the absorption of primary-beam $\mathrm{x}$-rays by the grid) may have on these images, it is important to remember that with focused grids this cutoff is most pronounced at the periphery of the grid and that grid lines would be virtually parallel in the centre. Thus, for radiography of the skull, the effect would be less apparent because of a relatively small, centrally placed region of interest. In the current work, all images assessed were found to be diagnostically acceptable, thus suggesting that the acquisition of images at increased SIDs with the appropriate grids would produce images of equally acceptable image quality or perhaps of increased quality because of the reduction of geometric unsharpness at the longer SID [36]. Nonetheless, a recommendation for clinical implementation is that a suitable radiographic grid is acquired to facilitate imaging at an increased SID. A limitation of the current study and a recommendation for future work would be to use image quality test tools in the methodology to acquire an objective measure of image detail. These data could then be compared with the results from the VGA evaluation panel. The use of pediatric phantoms is also recommended for future work to investigate the potential of increased SID in reducing the dose for pediatric cranial examinations.

When considering the implications of the inverse square law on increased SIDs for radiographic projections, it is important to address how this would affect the clinical situation. In accordance with the inverse square law, if a radiograph is acquired at a longer SID, then there is the potential to reduce the dose by a factor of four. In terms of image quality, this would result in a radiograph with increased noise unless the automatic exposure control device of the $\mathrm{x}$-ray set compensates for this by increasing the exposure time $(\mathrm{mA})$ or the exposure factors are manually adjusted by the radiographer. Work performed by Robinson and McLean [7] showed that when increasing the SID an adjustment factor can be applied to the $\mathrm{mA}$ value of the original SID to establish an appropriate $\mathrm{mA}$ value for the experimental SID. This adjustment factor (AF) was also used by Poletti and McLean and can be calculated using the following formula [14]:

$$
A F=\frac{\left(S I D_{\text {new }}\right)^{2}}{\left(S I D_{\text {old }}\right)^{2}}
$$

Provided that the entrance dose at the original SID is known, this AF can then be used to calculate the dose at a position (DP) corresponding with the entrance surface of a patient or the dose to the image receptor, and this equation is as follows:

$$
D P \text { new }=\frac{\left(D P_{\text {old }}\right)(A F)\left(S O D_{\text {old }}\right)^{2}}{\left(S O D_{\text {new }}\right)^{2}}
$$

where SOD is the source to object distance. 
In both diagnostic radiography and radiation therapy, the inverse square law is used to reduce the radiation dose at the entrance surface of a patient. If the DP is at the surface entry point, by increasing the SID while maintaining the same dose to the image receptor, the skin dose (ESD) will be reduced. The following example shows the practical application of equation 2 .

If an object $30 \mathrm{~cm}$ in diameter is imaged at a SID of $100 \mathrm{~cm}$ and the DP is known to be $40 \mathrm{mGy}$, then an estimated value can be calculated for the DP of the object imaged at a SID of $150 \mathrm{~cm}$ by using equations 1 and 2

$$
\begin{aligned}
& \text { If } A F=\frac{(150 \mathrm{~cm})^{2}}{(100 \mathrm{~cm})^{2}}=2.25 \\
& \text { Then DP } 150=\frac{(40 \mathrm{mGy})(2.25)(70 \mathrm{~cm})^{2}}{(120 \mathrm{~cm})^{2}} \\
& =30.625 \mathrm{mGy}
\end{aligned}
$$

These calculations predict an estimated dose reduction of more than 23\% when the SID is increased from 100 to $150 \mathrm{~cm}$. However, the calculations do not take into account the effect of $\mathrm{x}$-ray interactions with air or scattered radiation from the patient or object. By maintaining a constant exit dose at both SID extremes, the ESD acquired at each distance is a reasonable indicator of a dose change to the patient.

Radiography of the skull lends itself to the increased SID technique because both the $\mathrm{OF} 10^{\circ}$ and lateral skull projections are performed with a horizontal $\mathrm{x}$-ray beam in clinical practice [22]. Increasing the SID would not require additional bending or stretching by the radiographer because the tube would be travelling in the horizontal plane; thus, the implementation of this technique in clinical practice could be achieved with minimal discomfort to the radiographer. The research question posed at the beginning of this work was answered because the results show that increasing the SID is an effective optimization tool for both the OF $10^{\circ}$ and lateral skull projections imaged using a $\mathrm{dDR}$ system. The optimum SID for both projections was found to be $150 \mathrm{~cm}$ because this produced the greatest reduction in the effective dose $(21.9 \%$ for the OF $10^{\circ}$ skull projection and $23.9 \%$ for the lateral skull projection) and all images were found to be diagnostically acceptable. A variation exists in the literature in the manner in which the dose reductions are portrayed for other radiographic examinations, with some studies reporting ESD reductions and others reporting effective dose reductions. The level of dose reduction varies among authors, ranging from an $8.1 \%$ reduction for computer-simulated calculations [14] to a $44 \%$ reduction in the effective dose for patientoriented studies [6]. The reductions reported in the current work are comparable with reductions reported for pelvic examinations (ie, around $30 \%$ at $130 \mathrm{~cm}$ ) $[8,9]$.

The principle of increased SID may also be applied to other cranial examinations acquired with a horizontal beam such as facial bones, nasal bones, and sinuses. The European guidelines for $\mathrm{OF} 10^{\circ}$ skull projection (or PA skull projection) and lateral skull projection recommend $115 \mathrm{~cm}$ as a reference SID and consider a range of $100-150 \mathrm{~cm}$ as acceptable for this projection [25]. However, this research has found that a reference SID of $115 \mathrm{~cm}$ is too short an imaging distance, and that increasing the SID is a simple method of optimizing this examination. A recommendation of this research is that radiology departments and radiographers are shown the benefits of this technique with the view of introducing an updated reference SID of $150 \mathrm{~cm}$ into clinical practice. This could be introduced into the department by conducting experimental trials as part of the European Directive 97/43/Euratom clinical audit requirement. In Ireland, a requirement of this directive is that an external auditor appointed by the Health Service Executive conducts audits of radiologic practice every five years, and this incentive to review current techniques may assist the translation of research into practice for the increased SID technique.

\section{Conclusions}

This investigation concludes that increasing the SID from $100 \mathrm{~cm}$ is an effective optimization technique for $\mathrm{OF} 10^{\circ}$ and lateral cranial projections and thus should be considered for implementation in clinical practice. The optimum SID recommended for both projections is $150 \mathrm{~cm}$ because this SID yielded the greatest reduction in effective dose (ie, $21.9 \%$ for the $\mathrm{OF} 10^{\circ}$ skull projection and $23.9 \%$ for the lateral skull projection) while producing diagnostically acceptable images. In clinical practice, both examinations are performed using a horizontal $\mathrm{x}$-ray beam, thus minimizing the ergonomic limitations that may otherwise hinder implementation of the technique. Future research in this area will investigate the feasibility of implementing an increased SID in clinical practice and investigate the potential of an increased SID in reducing the dose for pediatric cranial examinations.

\section{References}

[1] Martin, C. J. (2008). Radiation dosimetry for diagnostic medical exposures. Radiat Prot Dosimetry 128, 389-412.

[2] ICRP. (2007). Publication 103, Recommendations of the International Commission on Radiological Protection. Ann ICRP 37, 2-4.

[3] Liu, S.-Z. (2010). Biological effects of low level exposures to ionizing radiation: theory and practice. Hum Exp Toxicol 29, 275-281.

[4] Uffmann, M., \& Schaefer-Prokop, C. (2009). Digital radiography: the balance between image quality and required radiation dose. EurJ Radiol 72, 202-208.

[5] Grondin, Y., Matthews, K., \& McEntee, M., et al. (2004). Dosereducing strategies in combination offers substantial potential benefits to females requiring X-ray examination. Radiat Prot Dosimetry 108, $123-132$.

[6] Brennan, P. C., \& Nash, M. (1998). Increasing FFD: an effective dose-reducing tool for lateral lumbar spine investigations. Radiography 4, 251-259.

[7] Robinson, J., \& McLean, D. (2001). Extended focal-film distance technique: an analysis of the factors in dose reduction for the AP knee radiograph. Radiography $7,165-170$.

[8] Brennan, P. C., McDonnell, S., \& O'Leary, D. (2004). Increasing film-focus distance (FFD) reduces radiation for X-ray examinations. Radiat Prot Dosimetry 108, 263-268. 
[9] Woods, J., Messer, S. (2009). Focussing on dose. Synergy September Issue, 16-20.

[10] Heath, D., England, A., \& Ward, A., et al. (2011). Digital pelvic radiography: increasing distance to reduce dose. Radiol Technol 83, 20-28.

[11] Dilger, R., Egan, I., \& Hayek, R. (1997). Effects of focus film distance (FFD) variation on entrance testicular dose in lumbar-pelvic radiography. Australas Chiropr Osteopathy 6, 18-23.

[12] Humphreys, S. (2003). Increasing FFD for high doses radiographic examinations and the effect on image quality. Radiography Ireland 7 , 211-213.

[13] Kebart, R. C., \& James, C. D. (1991). Benefits of increasing focal film distance. Radiol Technol 62, 434-442.

[14] Poletti, J. L., \& McLean, D. (2005). The effect of source to imagereceptor distance on effective dose for some common X-ray projections. Br J Radiol 78, 810-815.

[15] Australian Government Department of Human Services. Medicare Benefits Schedule (MBS) 2012. Available at: http://www.medicareaustralia. gov.au/provider/medicare/mbs.jsp. Accessed June 1, 2013.

[16] National Institute for Health and Clinical Excellence (2007). Head injury: triage, assessment, investigation and early management of head injury in infants, children and adults. NICE Clinical Guideline 56(4), National Health Service, UK

[17] Stiell, I. G., Clement, C. M., \& Rowe, B. H., et al. (2005). Comparison of the Canadian CT Head Rule and the New Orleans Criteria in patients with minor head injury. JAMA 294, 1511-1518.

[18] Hart, D., \& Wall, B. F. (2002). Radiation exposure of the UK population from medical and dental $\mathrm{x}$-ray examinations. Chilton: NRPB-W4.

[19] D'Sa, S., Abildgaard, N., Tighe, J., Shaw, P., \& Hall-Craggs, M. (2007). Guidelines for the use of imaging in the management of myeloma. Br J Haematol 137, 49-63.

[20] Freitas, M. B., \& Yoshimura, E. M. (2009). Diagnostic reference levels for the most frequent radiological examinations carried out in Brazil. Rev Panam Salud Publica 25, 95-104.

[21] Kay, A., \& Teasdale, G. (2001). Head Injury in the United Kingdom. World J Surg 25, 1210-1220.

[22] Sandstrom, S. (2003). The WHO manual of diagnostic imaging: radiographic technique and projections. Geneva: World Health Organisation in collaboration with the International Society of Radiology.

[23] Whitley, A. S., Sloane, C., Hoadley, G., Moore, A. D., \& Alsop, C. W (2005). Clark's positioning in radiography, (12th ed.). (pp. 517) London: Hodder Arnold.
[24] Bontrager, K. L., \& Lampignano, J. P. (2005). Radiographic positioning and related anatomy, (6th ed.). Philadelphia: Elsevier Mosby.

[25] European Commission (1996). European guidelines on quality criteria for diagnostic radiographic images. Luxembourg: Office for Official Publications of the European Communities.

[26] Tapiovaara, M., Lakkisto, M., \& Servomaa, A. (2008). PCXMC: a PC-based Monte Carlo program for calculating patient doses in medical X-ray examinations. Helsinki: Finnish Centre for Radiation and Nuclear Safety.

[27] Loubele, M., Bogaerts, R., \& Van Dijck, E., et al. (2009). Comparison between effective radiation dose of CBCT and MSCT scanners for dentomaxillofacial applications. Eur J Radiol 71, 461-468.

[28] Foley, S. J., McEntee, M. F., Achenbach, S., Brennan, P. C., Rainford, L. S., \& Dodd, J. D. (2011). Breast surface radiation dose during coronary CT angiography: reduction by breast displacement and lead shielding. AJR Am J Roentgenol 197, 367-373.

[29] Hansson, J., Båth, M., \& Håkansson, M., et al. (2005). An optimisation strategy in a digital environment applied to neonatal chest imaging. Radiat Prot Dosimetry 114, 278-285.

[30] Bath, M., \& Mansson, L. G. (2007). Visual grading characteristics (VGC) analysis: a non-parametric rank-invariant statistical method for image quality evaluation. BrJ Radiol 80, 169-176.

[31] Gorham, S., Brennan, P.C. Impact of focal spot size on radiologic image quality: a visual grading analysis. Radiography $16,304-313$.

[32] Hemdal, B., Andersson, I., \& Grahn, A., et al. (2005). Can the average glandular dose in routine digital mammography screening be reduced? A pilot study using revised image quality criteria. Radiat Prot Dosimetry 114, 383-388.

[33] Kelly, S., Berry, E., \& Roderick, P., et al. (1997). The identification of bias in studies of the diagnostic performance of imaging modalities. $\mathrm{BrJ}$ Radiol 70, 1028-1035.

[34] Brennan, P. C., McEntee, M., Evanoff, M., Phillips, P., O'Connor, W. T., \& Manning, D. J. (2007). Ambient lighting: effect of illumination on soft-copy viewing of radiographs of the wrist. $A J R$ Am J Roentgenol 188, W177-W180.

[35] Pallant, J. (2007). SPSS survival manual: a step-by-step guide to data analysis using SPSS for Windows, (3rd ed.). England: Open University Press, McGraw Hill Education.

[36] Graham, D. T., \& Cloke, P. (2003). Principles of radiological physics, (4th ed.). (pp. 12-15) Edinburgh: Churchill Livingstone.

[37] Butler, P., Mitchell, A. W. M., \& Ellis, H. (2006). Applied radiological anatomy. Cambridge: Cambridge University Press. 